\section{EPITHELIAL BIOLOGY}

\section{Cryptic migration}

Intestinal epithelial cells undergo high turnover, which is important for intestinal epithelium homeostasis and function. This continual cell renewal depends on the activity of stem cells that reside at the crypt base, which generate a highly proliferative population

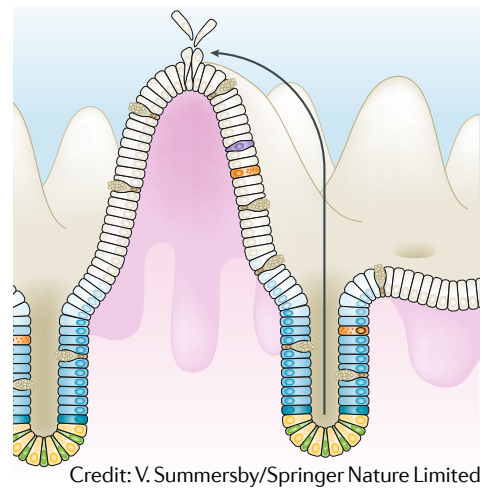
of cells that progressively differentiate while moving upwards along the villi, to be shed at the villi tips a few days later. It has been proposed that this epithelial migration along the villi is passive and driven by pushing forces generated by dividing stem cells. Krndija et al. now demonstrate the role of active collective cell migration in this process.

Short-term inhibition of proliferation does not block epithelial cell migration along the villi, leading the authors to hypothesize the involvement of active migration. Measuring cell density along the villi in mouse small intestines revealed an initial decrease in cell numbers starting from the crypt, followed by a gradual increase in the upper region of the villi. According to a biophysical model of intestinal epithelium dynamics that the authors developed, this cell density profile indicates a two-tier model of cell migration in the intestinal epithelium, whereby both stem cell division-derived forces (close to the crypt) and active cell migration (along the villi) are involved.

Live-cell imaging of mouse intestinal explants showed that cells maintained their cohesiveness when moving along the villi, indicating that they migrate collectively. Moreover, the measured mechanical tension in the epithelial sheet was highest at the back of the sheet (at the bottom of the villi), which is consistent with tensile stresses observed in collectively migrating epithelial sheets in vitro.

Super-resolution microscopy further revealed that epithelial cells moving along the villi extend - at their basal surface - small, actin-based protrusions that point towards the direction of movement. Short-term inhibition of the Arp2/3 complex - which nucleates branched actin networks important for cellular extensions - disrupted the polarity of these basal protrusions, reduced intra-epithelial tension and hindered cell migration. The inhibition of cell migration was more pronounced in the upper part of the villi, in accordance with the proposed two-tier model of epithelial cell migration in the intestine.

Thus, active collective cell migration contributes to intestinal epithelial turnover. It will now be important to understand how this active cell migration is regulated (for example, in terms of directionality) and whether its deregulation might lead to intestinal dysfunction.

Paulina Strzyz

ORIGINAL ARTICLE Krndija, D. et al. Active cell migration is critical for steady-state epithelial turnover in the gut. Science 365, 705-710 (2019)

\section{Journal club}

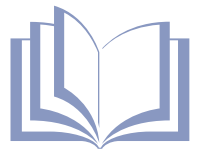

\section{EMIL HEITZ, A TRUE EPIGENETICS PIONEER}

The discovery of transposons by Barbara McClintock is a foundation of epigenetics. Transposons and their epigenetic modifications define non-transcribed regions of the genome termed heterochromatin, and their deregulation is associated with cancer. The origin of the term heterochromatin has become obscure - it was defined by Emil Heitz (1892-1965) in the first half of the 20th century.

In 1928, Heitz improved a cytological staining method and discovered that certain parts of mitotic chromosomes are more densely stained than others, thereby defining heterochromatin and euchromatin, respectively. The work was performed in liverworts, of which a model organism is Marchantia polymorpha. The compartmentation of chromatin was documented by Heitz in $>70$ species of bryophytes and in $>70$ species of flowering plants.

Research by Thomas Morgan on heredity encouraged Heitz to extend his investigation of heterochromatin to insects. In 1932 and 1933 he showed in plants and animals that sex chromosomes comprise more heterochromatin than autosomes do. With $\mathrm{H}$. Bauer he also identified the nature of polytene chromosomes, with their specific pattern of heterochromatin, in march flies, a discovery that later had a great impact on defining the nature of genes in fruit flies.

As early as 1929, Heitz proposed that heterochromatin is enriched in parts of chromosomes that do not carry genes or is associated with genetically 'passive' regions; this is still the basis of the contemporary definition of heterochromatin. The work of Heitz led to outstanding advances in understanding the links between genetics, development and heredity at a time when the molecular nature of the heredity material was still unclear.

Despite these contributions, Heitz is largely unknown and he is hardly ever mentioned in chromatin and epigenetics meetings. In part this is because his work was published in German and has never been translated. His obscurity could also be attributed to his dismissal from his professorship at the University of Hamburg in 1937, because his maternal grandfather was of Jewish descent. The dismissal disrupted his research for nearly 15 years and, despite a new appointment in Tübingen in 1952, Heitz did not resume his work on chromatin.

The unfortunate trajectory of the career of Emil Heitz can teach us at least two lessons: key findings are often made in studies of non-model organisms and the increasing feasibility of genome sequencing should trigger funding for research in such organisms; and the fate of Heitz in 1930s' Germany should alert us to the dangers of current nationalistic tendencies, which are fundamentally incompatible with science.

Frédéric Berger

Gregor Mendel Institute, Vienna, Austria email:Frederic.berger@gmi.oeaw.ac.at

The author declares no competing interests.

ORIGINAL ARTICLES Heitz, E. Das Heterochromatin der Moose. I. Jahrb. Wiss. Bot. 69, 762-818 (1928)| Heitz, E. Gescleschtschromosomen bei einem Laubmoos (Vorläufige Mitteilung). Ber. Dtsch. Bot. Ges. 50, 204-206 (1932) | Heitz, E. Uber totale und partielle somatische Heteropyknose, sowie strukturelle Gescleschtschromosomen bei Drosophila funebris (Cytologische Untersuchungen an Dipteren, II). Z.Zellforsch. Mikrosk. Anat. 19, 720-742 (1933) | Heitz, E. \& Bauer, H. Beweise für die Chromosomennatur der Kernschleifen in den Knäuelkernen von Bibio hortulanus L. (Cytologische Untersuchungen an Dipteren, I). Z.Zellforsch. Mikrosk. Anat. 17, 67-82 (1933)|Heitz, E. Heterochromatin, Chromocentren, Chromomeren (Vorläufige Mitteilung). Ber. Dtsch. Bot. Ges. 47, 274-284 (1929) 